\title{
Reinfarction During Hospitalization
}

National Cancer Institute

\section{Source}

National Cancer Institute. Reinfarction During Hospitalization. NCI Thesaurus. Code C80480.

Clinical diagnosis of a repeated infarction of an organ during a hospitalization. 